\title{
Back bombardment for dispenser and lanthanum hexaboride cathodes
}

\author{
Mahmoud Bakr, ${ }^{*}$ R. Kinjo, Y. W. Choi, M. Omer, K. Yoshida, S. Ueda, M. Takasaki, \\ K. Ishida, N. Kimura, T. Sonobe, T. Kii, K. Masuda, and H. Ohgaki \\ Institute of Advanced Energy, Kyoto University, Gokasho, Uji, Kyoto 611-0011, Japan
}

H. Zen

UVSOR, Institute for Molecular Science, Okazaki, Aichi 444-8585, Japan

(Received 21 December 2010; published 22 June 2011)

\begin{abstract}
The back bombardment (BB) effect limits wide usage of thermionic rf guns. The BB effect induces not only ramping-up of a cathode's temperature and beam current, but also degradation of cavity voltage and beam energy during a macropulse. This paper presents a comparison of the $\mathrm{BB}$ effect for the case of dispenser tungsten-base (DC) and lanthanum hexaboride $\left(\mathrm{LaB}_{6}\right)$ thermionic rf gun cathodes. For each, particle simulation codes are used to simulate the BB effect and electron beam dynamics in a thermionic rf gun cathode. A semiempirical equation is also used to investigate the stopping range and deposited heat power of BB electrons in the cathode material. A numerical simulation method is used to calculate the change of the cathode temperature and current density during a single macropulse. This is done by solving two differential equations for the rf gun cavity equivalent circuit and one-dimensional thermal diffusion equation. High electron emission and small beam size are required for generation of a high-brightness electron beam, and so in this work the emission properties of the cathode are taken into account. Simulations of the BB effect show that, for a pulse of $6 \mu \mathrm{s}$ duration, the DC cathode experiences a large change in the temperature compared with $\mathrm{LaB}_{6}$, and a change in current density 6 times higher. Validation of the simulation results is performed using experimental data for beam current beyond the gun exit. The experimental data is well reproduced using the simulation method.
\end{abstract}

DOI: 10.1103/PhysRevSTAB.14.060708

PACS numbers: $79.40 .+\mathrm{z}$

\section{INTRODUCTION}

Over the past few decades, the development of linear electron accelerator (linac) technology has been guided by increasing requirements on the quality of the electron beam. A bright electron beam with small emittance is indispensable for carrying out scientific investigations and solving numerous applied problems. An rf electron gun has features of compactness, inexpensive and easy handling compared with electrostatic guns. The high electric field in the rf gun cavity can be used to accelerate emitted electrons quickly to the state of $v \approx c$ (light velocity) [1]. Unlike the photocathode rf gun, the thermionic rf gun does not require unique and expensive laser devices. Generally speaking, rf gun apparatus are considered one of the highest quality sources for supplying electron beams with a desired beam energy and current [2]. An rf gun using thermionic and photocathodes was used for the first time at Stanford University [3] and Los Alamos National Laboratory [4], respectively. The thermionic rf gun has been chosen as electron injector for Kyoto University

\footnotetext{
*m-a-bakr@iae.kyoto-u.ac.jp
}

Published by the American Physical Society under the terms of the Creative Commons Attribution 3.0 License. Further distribution of this work must maintain attribution to the author(s) and the published article's title, journal citation, and DOI.
Free Electron Laser facility (KU-FEL) [5] due to the high quality of its electron beam. On the other hand, the thermionic rf gun has its own characteristic problem, which is the change of cathode temperature and current density during a macropulse. Since electrons are accelerated in only one half of an rf cycle, electrons emitted during the other half cycle are unable to escape the gun before the electric field changes direction. These electrons are referred as back-streaming electrons. These electrons hit the cathode surface, causing an increase in cathode temperature and the ramping-up of emission current density during the macropulse. As a result, the beam quality and FEL stability are seriously affected. Many scientific publications deal with reducing back bombardment (BB) effects in thermionic rf guns. Most of these papers consider modifications of the gun design or the cavity field [5-11], however only a few papers have focused on the role of the cathode material itself.

The KU-FEL thermionic rf gun consists of a 4.5-cell cavity driven by a $10 \mathrm{MW}$ rf source, providing electron beams of energy up to $10 \mathrm{MeV}$ with a total gun length of $30 \mathrm{~cm} \mathrm{[5].} \mathrm{A} \mathrm{thermionic} \mathrm{cathode} \mathrm{is} \mathrm{set} \mathrm{on} \mathrm{a} \mathrm{flat} \mathrm{surface} \mathrm{at}$ the center of a cylindrically symmetric half cell in the gun cavity. The cathode of the KU-FEL rf gun has been improved since 2007, where the BB effect made it difficult to produce high beam currents and energies for macropulses longer than $1 \mu \mathrm{s}$. We have successfully produced 
higher beam currents over longer macropulses by substituting the dispenser (DC) tungsten-base cathode (W-BaO/CaO/ $/ \mathrm{Al}_{2} \mathrm{O}_{3}$ ) with a single crystal of $\mathrm{LaB}_{6}$. The performance of the KU-FEL rf gun has thus been dramatically improved, however BB in the cathode still affects the macropulse duration below $6 \mu \mathrm{s}$ [12].

Simulation is the best way to study the BB effect in a thermionic rf gun. To clarify the $\mathrm{BB}$ phenomenon for future improvement, in the present paper we compare $\mathrm{BB}$ effects between the $\mathrm{DC}$ and $\mathrm{LaB}_{6}$ cathodes by investigating heating properties of the cathode materials. In the first section, the materials used in the simulation are introduced. The second section is devoted to the numerical calculation for thermionic emission, transverse emittance, stopping range, and deposited heat power for $\mathrm{DC}$ and $\mathrm{LaB}_{6}$ cathodes. The change of the cathode temperature and current density are then determined. Simulation results and their comparison with experimental data for the $\mathrm{LaB}_{6}$ cathode are presented in the third section. The fourth section presents an overall discussion of the simulation and demonstration results, and the final section presents our conclusions.

\section{CATHODE MATERIALS}

We are interested in the choice of appropriate cathode material for reducing the effect of BB. Towards this end, it is important to clarify the factors causing variation of cathode temperature and current density. The temperature variation caused by $\mathrm{BB}$ electrons during the macropulse is determined by the penetration depth of electrons into the cathode material and, consequently, by the energy spectrum of electrons [13]. It is reported that, for an rf gun cathode with typical beam characteristics, the minimum emission parameters required for cathode at field strength of 30-50 $\mathrm{MV} \mathrm{m}^{-1}$ and pulse length up to $10 \mu \mathrm{s}$ are [13]: (i) high current density (more than $20 \mathrm{~A} \mathrm{~cm}^{-2}$ ), which allows for the design of small cathode sizes (diameter of $1.5-2 \mathrm{~mm}$ ) and the potential for obtaining a low emittance electron beam; (ii) high resistance of the cathode to sputtering; and (iii) small sublimation rate of emitting substrate (not more than $10^{-6} \mathrm{~kg} \mathrm{~m}^{-2} \mathrm{~s}^{-1}$ ).

Because of their inherent ability to emit current of high density, DC (impregnated) has been used as an electron emitter long before the usage of hexaborides. The impregnation is important because it is considered to be the source of barium needed to obtain a low work function surface. DCs are commonly impregnated by placing the oxidized barium calcium aluminate impregnates in contact with the emitting surface of a porous W (tungsten) structure and then heating the assembly past the melting point of the impregnate material to about $1923 \mathrm{~K}$. Reliable electron current over a long period of time is a function of the equilibrium established between the rate of arrival of barium at the emitting surface and the rate of evaporation of barium from the emitting surface [14]. DC was used as an
TABLE I. DC and $\mathrm{LaB}_{6}$ parameters used in the comparison.

\begin{tabular}{|c|c|c|}
\hline & DC & $\mathrm{LaB}_{6}$ \\
\hline Molecular weight: $\mathrm{g} \mathrm{mol}^{-1}$ & 183.85 & 203.772 \\
\hline Density: $\mathrm{kg} \mathrm{m}^{-3}$ & 15440 & 4720 \\
\hline Melting temperature: $\mathrm{K}$ & 1923 & 2483 \\
\hline Thermal conductivity: $\mathrm{Wm}^{-1} \mathrm{~K}^{-1}$ & 174 & 147 \\
\hline Specific heat: $\mathrm{J} \mathrm{kg}^{-1} \mathrm{~K}^{-1}$ & 132 & 122 \\
\hline Richardson constant: $\mathrm{A} \mathrm{cm}^{-2} \mathrm{~K}^{-2}$ & 1.5 & 29 \\
\hline Work function: eV & 1.56 & 2.66 \\
\hline Effective atomic number & 74 & 40.447 \\
\hline Effective molecular weight: $\mathrm{g} \mathrm{mol}^{-1}$ & 183.85 & 94.735 \\
\hline
\end{tabular}

electron emitter for many facilities over the world. In KU-FEL, a DC was used as cathode material until its substitution with a single crystal of $\mathrm{LaB}_{6}$ in 2007.

Alkaline-earth metals, rare earth metals, and thorium form of borides of the type $\mathrm{MB}_{6}$, all have the same cubic crystal structure. The small boron atoms form a threedimensional framework structure which surrounds the large metal atom. The properties of $\mathrm{MB}_{6}$ compounds suggest they may be an excellent choice of cathode material. These include low work function, low volatility, low electrical resistivity, high mechanical strength, and high chemical resistance. The borides are characterized by high melting temperatures, and most of them have high thermal conductivity. Further characteristics are a fair corrosion resistance, chemical inertness, good wear resistance, and a thermal shock resistance much better than that of oxide ceramics [15]. The most promising hexaborides to be used as electron emitters are $\mathrm{LaB}_{6}$ and $\mathrm{CeB}_{6}$ which have been intensively studied since Lafferty in the $1950 \mathrm{~s}$. $\mathrm{LaB}_{6}$ has been selected to replace DC as thermionic cathode in many FEL facilities across the entire world. Table I lists the most essential physical and chemical properties for materials under investigation. Because of the difficulty of determining some DC parameters like atomic and molecular weights, thermal conductivity, and specific heat, the corresponding parameters of pure tungsten are assumed. Density, melting temperature, work function, and Richardson's constant for DC and $\mathrm{LaB}_{6}$ are picked from Refs. [14,15].

\section{NUMERICAL CALCULATIONS}

Here we present the model for studying the effect of BB electrons on $\mathrm{DC}$ and $\mathrm{LaB}_{6}$ cathodes. The modeling sequence begins by determining the emission properties of the materials. The stopping range and deposited heat power of $\mathrm{BB}$ electrons are then determined. Finally, a numerical simulation model is developed to calculate the change in cathode temperature and current density during a macropulse.

\section{A. Thermionic emission}

The most important parameter controlling electron emission in a thermionic cathode is the surface work 
function, which is related to the current density $J_{c}$ $\left(\mathrm{A} \mathrm{cm}^{-2}\right)$ through the Richardson equation:

$$
J_{c}=A T^{2} \exp \left(-\frac{e \phi}{k_{B} T}\right),
$$

where $A\left(\mathrm{~A} \mathrm{~cm}^{-2} \mathrm{~K}^{-2}\right)$ denotes Richardson's constant, $e$ (C) is the electron charge, $k_{B}\left(1.38 \times 10^{-23} \mathrm{JK}^{-1}\right)$ is Boltzmann's constant, $T(\mathrm{~K})$ is the cathode temperature, and $\phi(\mathrm{eV})$ is the material work function (a material dependent property). A larger value for the work function implies the requirement of higher temperature and thus more cathode heater power for achieving the desired current density. When $\mathrm{BB}$ electrons hit a cathode during a macropulse, the cathode temperature and consequently $J_{c}$ increase. It is well known that the electron beam current exiting the gun is dependent on $J_{c}$. Thus, it is important to assign some criterion on current density for the present calculation. Let us consider the minimum required current density to be $20 \mathrm{~A} \mathrm{~cm}^{-2}\left(J_{\min }\right)$.

\section{B. Transverse emittance}

The transverse emittance $\varepsilon_{\text {rms }}(\pi \mathrm{mm}$ mrad) of an electron beam on the cathode as a function of its temperature and radius can be defined as [16]

$$
\varepsilon_{\mathrm{rms}}=\frac{r_{c}}{2} \sqrt{\frac{k_{B} T}{m_{e} c^{2}}} .
$$

where $r_{c}(\mathrm{~mm})$ is a cathode radius, $m_{e}(\mathrm{~kg})$ the electron mass, and $c\left(\mathrm{~ms}^{-1}\right)$ is the speed of light. It is well known that in practice, thermionic rf guns do not achieve emittances as small as the ideal theoretical emittance. Even so, a smaller emittance of several $\pi \mathrm{mm}$ mrad is recommended for FEL operation. In order to achieve this, the cathode diameter must be of order a few $\mathrm{mm}$ for temperatures in the range $1000-2500 \mathrm{~K}$.

\section{Stopping range and deposited heat power}

Electrons penetrating a material lose their energy through interaction with atoms in that material. Most of the electrons' kinetic energy is converted to thermal energy. This thermal energy is deposited inside the material layers, causing the material to heat up. The range of electrons inside a material is useful for evaluating the effects associated with deep penetration of electrons, such as BB ones. The extrapolated range or "stopping range" $(R)$ is usually defined as the thickness of material at which the extension of the linearly decreasing region of the transmission curve becomes zero. At low energies, $R$ is frequently determined from linear extrapolation of the energy distribution measured for a given thickness of the absorber. For absorbers of high atomic number, the transmission curve often does not show the linear region, and the extrapolation is then made using the tangent to the steepest point of the curve [17].
For electrons with energy from $0.3 \mathrm{keV}-30 \mathrm{MeV}$ in an absorber with atomic number $6-92, R$ can be expressed by a single semiempirical equation (TIO) of the form [17]

$$
\begin{aligned}
R & =\frac{a_{1}}{\rho}\left[\frac{\ln \left(1+a_{2} \tau\right)}{a_{2}}-\frac{a_{3} \tau}{1+a_{4} \tau^{a_{5}}}\right], \quad a_{1}=\frac{2.335 A}{Z^{1.209}}, \\
a_{2} & =1.78 \times 10^{-4} Z, \quad a_{3}=0.9891-\left(3.01 \times 10^{-4} Z\right) \\
a_{4} & =1.468-\left(1.180 \times 10^{-2} Z\right), \quad a_{5}=\frac{1.232}{Z^{0.109}},
\end{aligned}
$$

where $\rho\left(\mathrm{kg} \mathrm{m}^{-3}\right)$ is the absorber (cathode material) density, $\tau$ is the incident kinetic energy in the rest frame of electron, and the parameters $a_{i}(i=1,2, \ldots, 5)$ are given by a simple function of atomic number $Z$, and atomic weight $A$. In the case of a mixture or compound $Z$ and $A$ are replaced by the effective values of atomic number $\left(Z_{\text {eff }}\right)$ and effective atomic weight $\left(A_{\text {eff }}\right)$ as shown below:

$$
Z_{\mathrm{eff}}=\sum_{i} f_{i} Z_{i}, \quad A_{\mathrm{eff}}=\frac{Z_{\mathrm{eff}}}{(Z / A)_{\mathrm{eff}}}, \quad(Z / A)_{\mathrm{eff}}=\sum_{i} \frac{f_{i} Z_{i}}{A_{i}},
$$

where $f_{i}$ is the fraction by weight of the constituent element with $Z_{i}$ and $A_{i}$. The effective atomic number and the effective atomic weight for the $\mathrm{LaB}_{6}$ are listed in Table I. For DC we use the same values of pure tungsten as listed. The deposited heat of electrons inside the absorber is considered the main source of heating during a macropulse. This can be defined as the loss of electron energy $\Delta E(\mathrm{eV})$ in a material of infinitesimally small thickness $\Delta R$ $(\mu \mathrm{m})$, written $\Delta E / \Delta R,[17]$.

\section{Change of cathode temperature and current density}

The electron beam extracted from a thermionic rf gun consists of macropulse and micropulse structures. The micropulse structure is defined as the electron beam evolution over one rf period. This varies continuously and periodically as rf power is fed to a thermionic rf gun. Usually the macropulse duration is less than $10 \mu \mathrm{s}$ and its repetition rate is less than $100 \mathrm{~Hz}$ for infrared radiation sources generated by FEL. The BB electrons have the same pulse structure as the extracted beam, thus the heat input from $\mathrm{BB}$ electrons also has micropulse and macropulse structure. During a macropulse, the cathode temperature rapidly increases, and after the macropulse the cathode temperature gradually decreases. As a result, the temperature increase and decrease occur periodically. This causes an increase in the average cathode temperature until a balance in the cathode heating and cooling power (radiative and conductive) is reached. The average temperature increase is not a serious problem, and is easily controlled by adjusting the cathode heater [18]. On the other hand, the temperature rise during a macropulse cannot be compensated using this method.

In order to calculate the temperature increase accurately, it is necessary to consider the distribution of electron BB and the heat distribution at the cathode surface. We use a previously developed numerical simulation model [19], to 


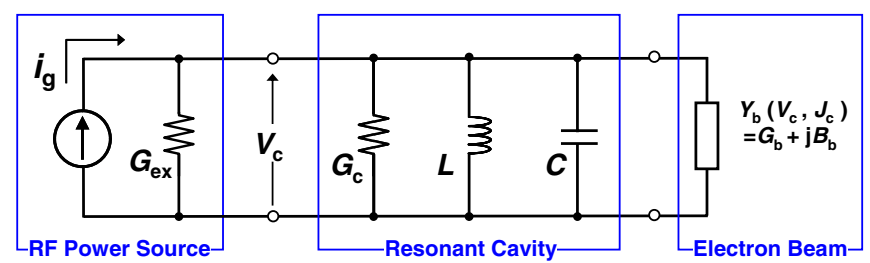

FIG. 1. Equivalent circuit of an rf gun consists of an $\mathrm{rf}$ power source, a resonant cavity, and a beam loading [20].

determine the ramping of cathode surface temperature and current density during the macropulse. The model simultaneously solves two differential equations. The first one is the differential equation of the $\mathrm{rf}$ gun cavity equivalent circuit [Eq. (5)], which corresponds to the circuit shown in Fig. 1 [20]. The differential equation for the rf gun equivalent circuit is given as

$$
\begin{aligned}
\frac{d I_{L}(t)}{d t}= & \frac{1}{L} V_{c}(t)-i 2 \pi f_{0} I_{L}(t) \\
\frac{d V_{c}(t)}{d t}= & \frac{1}{C}\left\{i_{g}(t)-Y_{b}\left(t, V_{c}, J_{c}\right) V_{c}(t)-I_{L}(t)(\beta+1) G_{c} V_{c}(t)\right\} \\
& -i 2 \pi f_{0} V_{c}(t) \\
C_{c}= & \frac{1}{2 \pi f_{0}(R / Q)}, \quad L_{c}=\frac{2 \pi f_{0}}{(R / Q)}, \quad G_{c}=\frac{1}{Q_{0}(R / Q)}, \\
G_{\mathrm{ex}}= & \beta G_{c},
\end{aligned}
$$

where $f_{0}(\mathrm{~Hz})$ denotes the resonant frequency of the $\mathrm{rf}$ resonant cavity, $Q_{0}$ the unloaded quality factor, $R / Q(\Omega)$ the $R / Q$ factor of the cavity, and $\beta$ the coupling coefficient of the input waveguide to the cavity. The source current $I_{g}$ is described as $I_{g}=\left(8 G_{\mathrm{ex}} P_{\mathrm{in}}\right)^{1 / 2}, P_{\mathrm{in}}$ is the input rf power, and $G_{\mathrm{ex}}$ is the external conductance. $L_{c}, C_{c}$, and $G_{c}$ are cavity inductance, capacitance, and conductance, respectively, with the values and details of these constants given in Ref. [19]. The beam admittance $Y_{b}$ is defined as $Y_{b}=$ $I_{b} / V_{c}$, where $I_{b}$ denotes the beam loading current and $V_{c}$ $\left(\mathrm{MV} \mathrm{m}^{-1}\right)$ is the cavity field. The latter is kept constant during experiment by modifying the input rf power pulse shape during the macropulse [11]. The beam admittance is divided into real and imaginary parts, which are called the beam conductance $G_{b}$ and beam susceptance $B_{b}$, respectively. The conductance and susceptance depend on energy consumed by an electron beam during the riding rf phase; however, this changes during a macropulse due to the changes in current density and, hence, the beam loading. In our calculations the beam conductance and beam susceptance are determined in advance by using KUBLAI code [21], which enables determination of the beam loading.

The second equation used in the numerical simulation model is the one-dimensional thermal diffusion equation for the thermionic cathode, which includes a heat input from BB electrons [Eq. (6)]:

$$
C \rho V \frac{\partial T(z, t)}{\partial t}=\lambda \frac{\partial^{2} T(z, t)}{\partial z^{2}}+Q_{b}\left(z, t, V_{c}, J_{c}\right),
$$

where $C\left(\mathrm{~J} \mathrm{~kg}^{-1} \mathrm{~K}^{-1}\right)$ denotes the specific heat capacity, $\lambda$ $\left(\mathrm{Wm}^{-1} \mathrm{~K}^{-1}\right.$ ) the thermal conductivity, $z(\mathrm{~m})$ the depth of cathode from the surface, $V\left(\mathrm{~m}^{3}\right)$ is the cathode volume, and $Q_{b}$ the heat input due to $\mathrm{BB}$ electrons. In the numerical model a flat rf pulse is used to show the effect of the current density increase. A simple explanation for the used model is presented as follows.

The cathode of thickness $2 \mathrm{~mm}$ is divided into 20000 thin disks. At the initial state before starting the BB effect, the heat input from the cathode heater and radiative heat loss on the cathode surface are balanced with each other. Thus, heat fluxes in each disk are the same and given by the radiative loss on the cathode surface. The heat flux for the first disk is prepared in advance by using particle simulation code, PARMELA 3.38 [22]. BB electrons lose their energy by penetrating the cathode disks; the lost energy depends on electron energy and the penetration depth. Therefore, every disk receives different energy and heats up with a different amount. The heat transfer between the disks is then determined by the difference method. Finally, the change in the disk temperature and corresponding change in the current density is calculated. Initial cathode temperatures supplied to the simulation model are $1476 \mathrm{~K}$ and $1981 \mathrm{~K}$ of $\mathrm{DC}$ and $\mathrm{LaB}_{6}$, respectively corresponding to $J_{\min }$. It is impossible to solve Eq. (6) analytically, since the heat input $Q_{b}$ is time dependent. For this reason the equation is solved using an iterative method. To investigate the change in the cathode temperature and current density an $8 \mathrm{MW}$ during $6 \mu$ s macropulse rf pulse duration is used in the simulation model.

\section{RESULTS OF THE SIMULATION, COMPARISON WITH EXPERIMENT}

We assume here that the simulated cathodes have the same conditions of vacuum level, heating method, applied electric field in the rf cavity, diameter, and area. Section IVA sheds light on some basic physical features of the BB process through simulating the motion of electrons in KU-FEL thermionic rf gun. Sections IV B and IV C compare the cathodes from a beam emission point of view. Section IVD shows the results of the stopping range and deposited heat power in $\mathrm{DC}$ and $\mathrm{LaB}_{6}$. Section IVE shows a determination of the change of cathode temperatures and the equivalent change in the current densities. Simulation results are compared to experimental data in section IV F.

\section{A. Picture for BB in thermionic rf gun}

In order to evaluate the effect of $\mathrm{BB}$ electrons on the time evolution of cathode temperature, the BB energy distribution and deposited heat are calculated. Results are shown in Fig. 2.

Figure 2(a) shows the BB electron energy distribution as a function of the cathode area. Figure 2(b) shows the BB energy spectrum as a function of electron kinetic energy at 

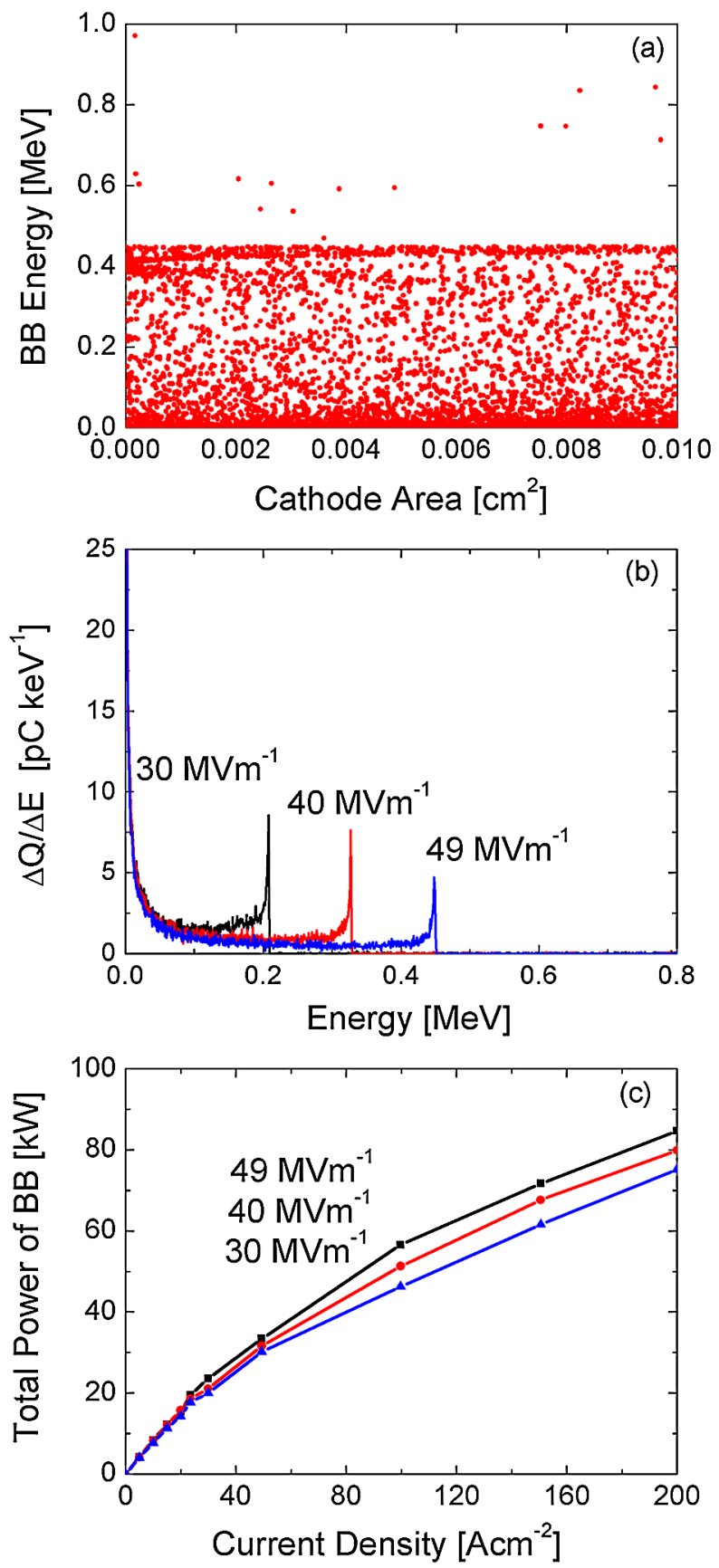

FIG. 2. (a) BB electron energies distribution as a function of the cathode area. (b) Energy spectrum of BB electrons at different values of cavity field. (c) BB total power as a function of current density at different values of cavity field.

different values of the cavity field. Figures 2(a) and 2(b) are both derived using the PARMELA code. Meanwhile, Fig. 2(c) shows the total BB power as a function of current density at different values of cavity field, as derived using the KUBLAI code.

It can be seen from Fig. 2(a) that BB has almost uniformed distribution as a function of the cathode area. Hence, there would be no significant effect of BB distribution neither in the cathode temperature nor in the beam

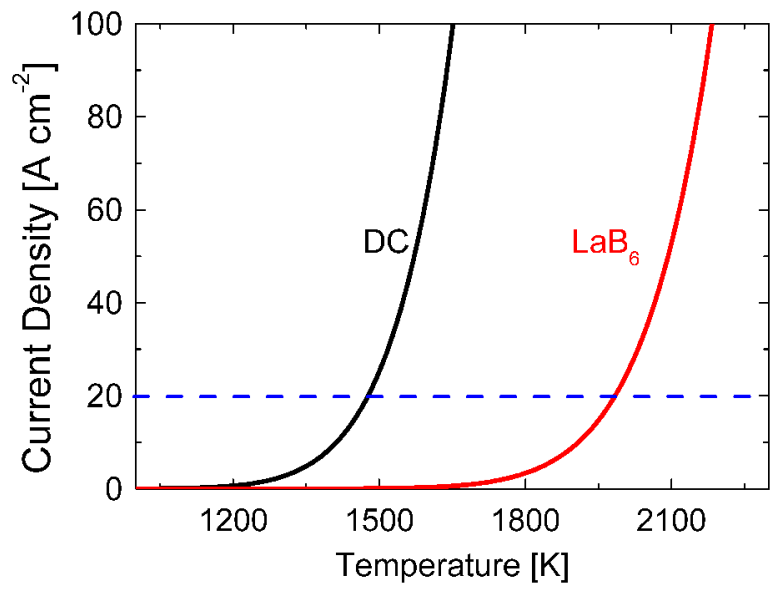

FIG. 3. Ideal emission of $\mathrm{DC}$ and $\mathrm{LaB}_{6}$ cathodes calculated using Eq. (1).

emittance. Meanwhile, Fig. 2(b) indicates that BB electrons hitting a cathode surface have an energy distribution that depends on the cavity field and current density on the cathode surface (i.e. the cathode temperature). The total power of BB electrons also depends on the cavity field and cathode current density as shown in Fig. 2(c). In general, from the above picture it can be understood that the total power of BB electrons increases when a thermionic rf gun is operated under conditions of high current density or cavity field.

\section{B. Thermionic emission}

The ideal behavior for thermionic emission from DC and $\mathrm{LaB}_{6}$ is calculated using Eq. (1) and shown in Fig. 3. It can be seen from Fig. 3 that both $\mathrm{DC}$ and $\mathrm{LaB}_{6}$ could emit $J_{\text {min }}$ before reaching their respective melting temperatures. Because of the small value of the work function for DC $(1.56 \mathrm{eV})$ compared to $\mathrm{LaB}_{6}(2.66 \mathrm{eV})$, the current density emitted from DC can satisfy $J_{\min }$ at a lower temperature of $1476 \mathrm{~K}$ (compare to $1981 \mathrm{~K}$ for $\mathrm{LaB}_{6}$ ).

\section{Transverse emittance}

The transverse emittance for $\mathrm{DC}$ and $\mathrm{LaB}_{6}$ is calculated using Eq. (2) and the results are listed in Table III. We consider cathodes to have radius $r_{c}=1 \mathrm{~mm}$. The temperatures used to calculate the transverse emittance are 1476 and $1981 \mathrm{~K}$ for DC and $\mathrm{LaB}_{6}$, respectively, corresponding $J_{\min }$. In practice, it is difficult to achieve such low emittance using our rf gun; however, it gives an indication for the minimum requirements from the cathode temperature and radius for FEL operations. Successfully, we could achieve lasing with a $\mathrm{LaB}_{6}$ cathode of $1 \mathrm{~mm}$ radius and the measured emittance in that time around $3 \pi \mathrm{mm} \mathrm{mrad} \mathrm{[23].}$

\section{Stopping range and deposited heat}

The stopping range and the deposited heat power for BB electrons having energy less than $1 \mathrm{MeV}$ (chosen based on 
the simulation results in Sec. IVA) are determined by using the TIO equation, Eq. (3). In the case of both $\mathrm{DC}$ and $\mathrm{LaB}_{6}$, the results are depicted in Fig. 4. The stopping range and heat deposited for the simulated materials are shown in Figs. 4(a) and 4(b), respectively. Meanwhile, Fig. 4(c) shows the deposited energy at specific BB electron energies $(100,300$, and $500 \mathrm{keV})$ for $\mathrm{DC}$ and $\mathrm{LaB}_{6}$. It is important to note that, for the case of DC, adjusting the
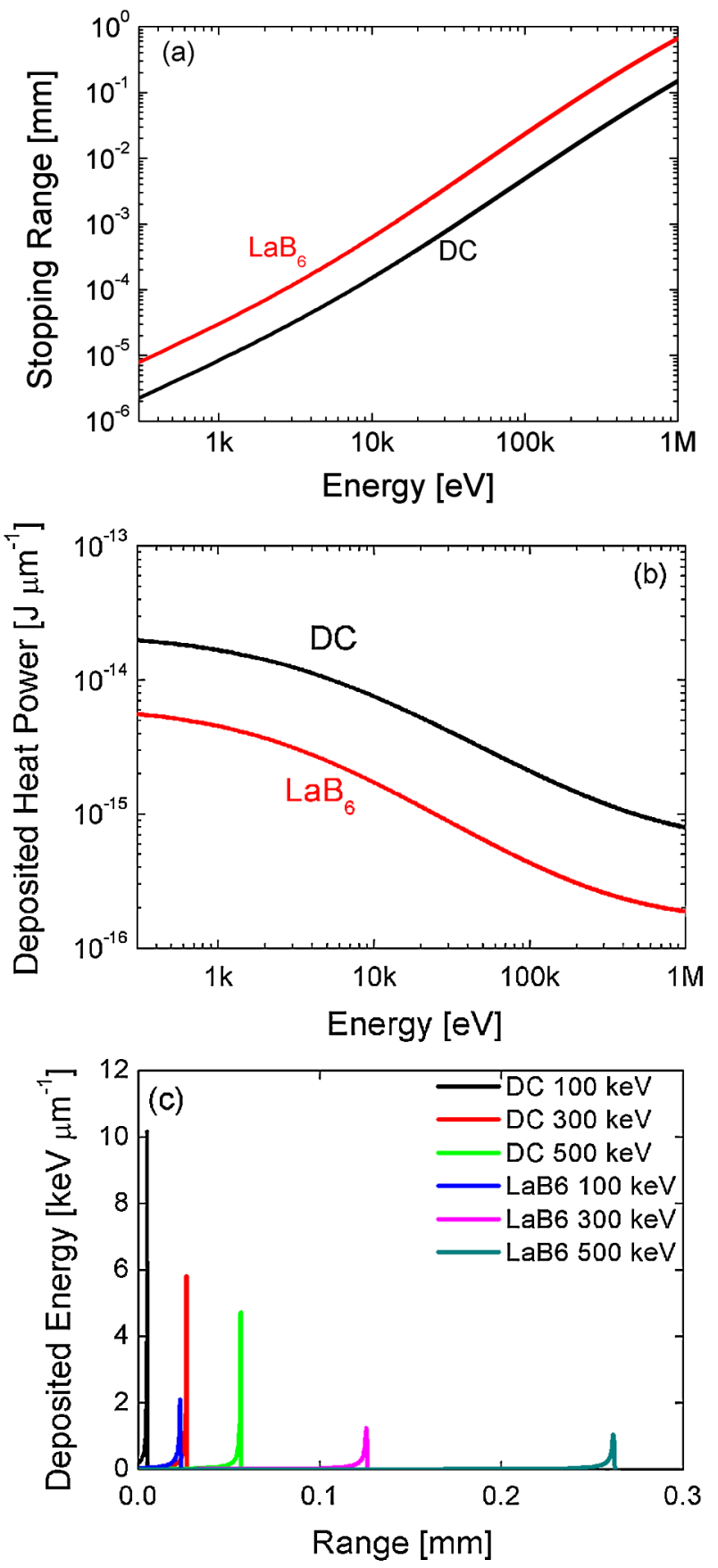

FIG. 4. Comparison of the stopping range (a) and the deposited heat power (b) as a function of BB electron energy. (c) The deposited energy at different $\mathrm{BB}$ electron energies as a function of the range in the cathode. effective atomic number and atomic weight to $80 \%$ of the pure tungsten values (as appropriate for its density) does not result in a significant difference to Fig. 4.

\section{E. Change of cathode temperature and current density}

The ramping-up of cathode temperature and current density due to heat deposited from $\mathrm{BB}$ for $\mathrm{DC}$ and $\mathrm{LaB}_{6}$ is determined using the numerical model mentioned previously. In Figs. 5(a) and 5(b) the changes in cathode temperature and current density during a pulse of $6 \mu \mathrm{s}$ duration with flat $8 \mathrm{MW}$ rf field are depicted, respectively. The initial values of temperature and current density which supplied to the simulation model were 1476 and $1981 \mathrm{~K}$ (corresponding to $J_{\min }$ ) for $\mathrm{DC}$ and $\mathrm{LaB}_{6}$, respectively. Initial parameters for the equivalent rf cavity circuit and cathodes properties are listed in Tables I and II.

\section{F. Comparison between numerical and experimental results}

First, for verification of the numerical simulation model, a comparison between the simulated and measured beam currents beyond the gun aperture is conducted. Here the
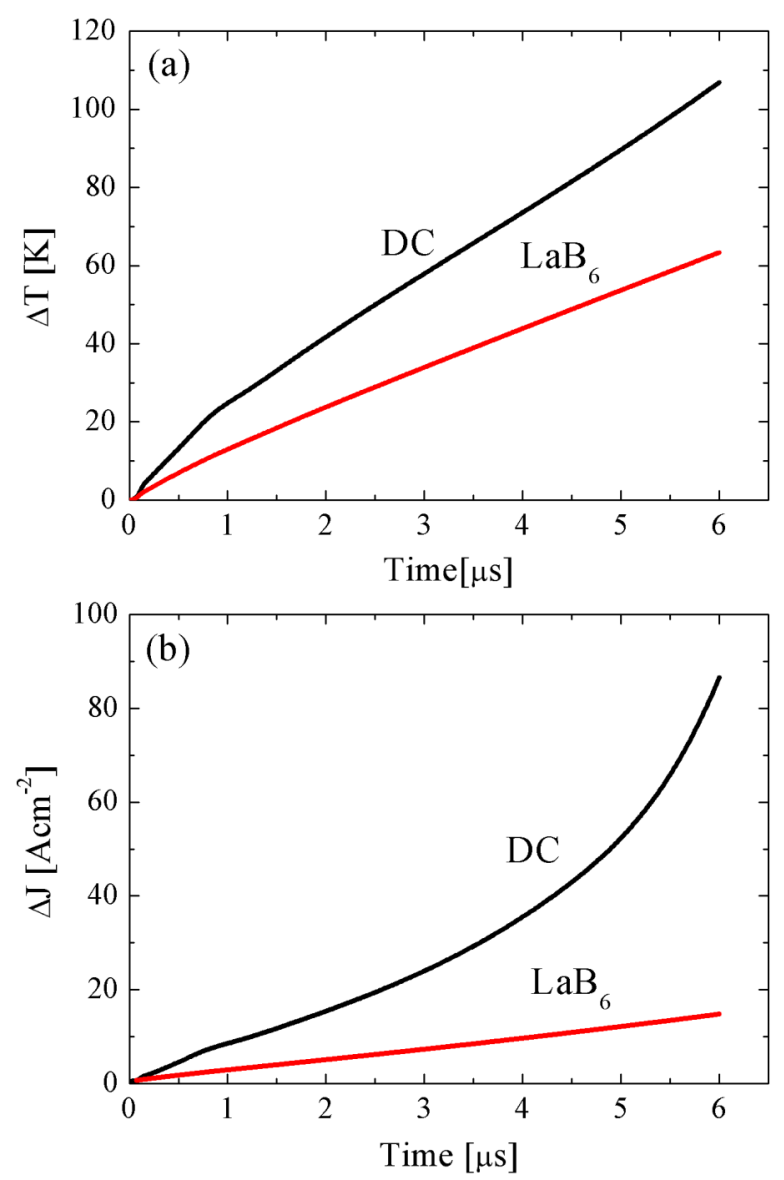

FIG. 5. Temporal evolutions of change in a cathode temperature (a) and current density (b) for $\mathrm{DC}$ and $\mathrm{LaB}_{6}$ calculate from the numerical model. 
TABLE II. Parameters of KU-FEL thermionic rf gun used in the simulation.

\begin{tabular}{lc}
\hline \hline Resonant frequency $[\mathrm{MHz}]$ & 2856 \\
Coupling coefficient $\beta$ & 2.79 \\
$Q$ value & 12500 \\
$R / Q[\Omega]$ & 980 \\
Accelerating mode & $\pi$ \\
Cathode radius $[\mathrm{mm}]$ & 1 \\
Current density $\left[\mathrm{A} \mathrm{cm}{ }^{-2}\right]$ & 20 \\
Macropulse duration $[\mu \mathrm{s}]$ & 6 \\
\hline \hline
\end{tabular}

conditions of the experimental and simulation results are introduced.

The experimental setup for measuring the beam current beyond the gun exit in the KU-FEL thermionic rf gun is shown in Fig. 6(a). In the first half cell, a $1 \mathrm{~mm}$ radius $\mathrm{LaB}_{6}$ cathode is mounted. A viewport is mounted on the half cell. Through the viewport, the cathode surface temperature can be measured by the use of an infrared (IR) thermometer, which is $0.2 \mathrm{~m}$ away from the cathode surface. A transverse magnetic field was applied around the cathode surface to reduce $\mathrm{BB}$ power.

During the experiment, the beam current was measured by a current transformer (CT1). Figure 6(b) shows measured time evolutions of the beam current together
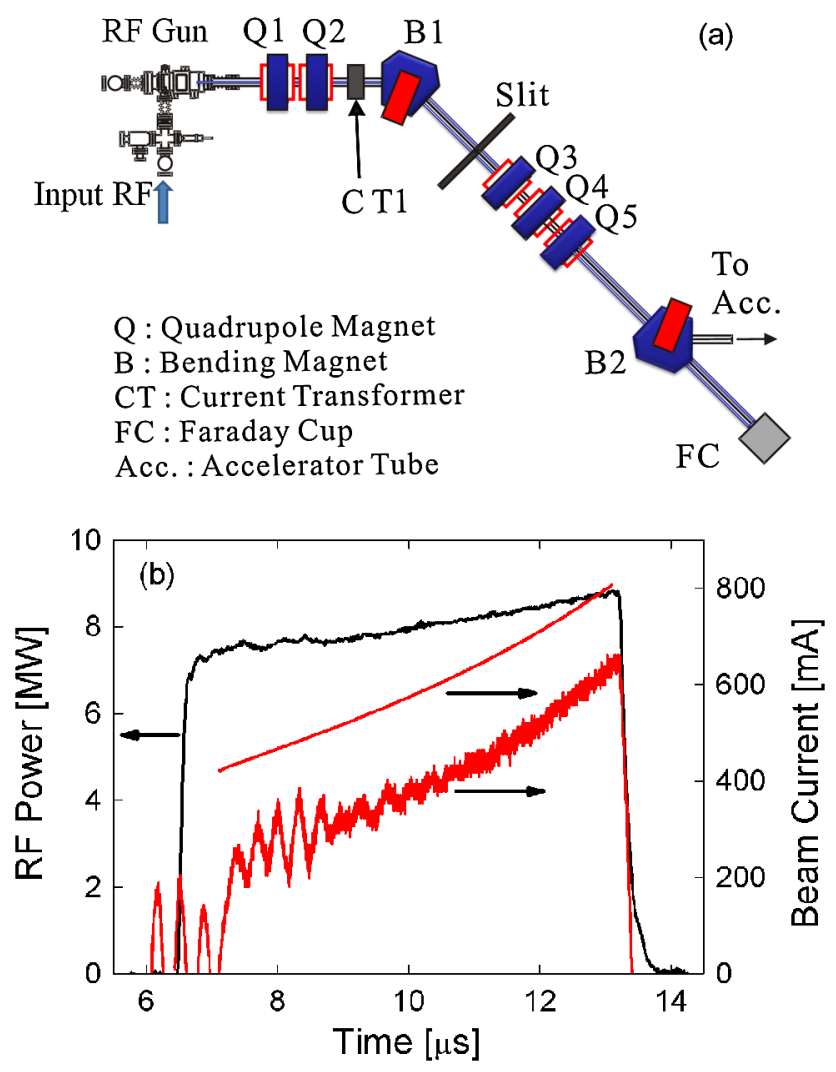

FIG. 6. Experimental setup for measuring beam current beyond the gun aperture (a). Experimental results for input rf power and beam current with simulation results (b). with the pulsed input rf power for a macropulse of $6 \mu \mathrm{s}$ duration. In the normal experimental conditions in our rf gun, the input rf pulse for the gun is usually modified with small slope to keep the cavity voltage at constant value [11]. The modified input rf power with resonant frequency of $2855.975 \mathrm{MHz}$ is used in the simulation model.

A numerical simulation was carried out to demonstrate the experimental results. All parameters used in the simulation are the same as those in the experiment. The initial cathode temperature is assumed to be $1650^{\circ} \mathrm{C}$ as for the experimental cathode, because for the measured cathode temperature has uncertainty. To compare the measured current with the calculated one, the current density determined from the simulation is multiplied over the cathode area. This is under the assumption that the extracted electron beam size at the cathode surface has the same area as the cathode itself. Simulation results of the beam current are depicted in Fig. 6(b).

As shown in Fig. 6(b), the experimental results are well reproduced by simulation. The vertical shift between the experimental and simulation results can be explained as arising from two factors. The first one is the loss of the electron beam in the trajectory from the cathode surface up to CT1 which is far from the cathode surface (around $1 \mathrm{~m}$ ). The second factor is the uncertainty in measuring cathode temperature using the IR detector due to the difficulty to determine the real temperature.

\section{DISCUSSION}

Research on high-brightness electron sources is now very important in order to widen the range of the radiation available for material science research and conduct new kinds of experiments, such as Compton scattering and electron beam collider, etc. From this point of view, a high-brightness electron source is required; this implies a high peak current (10-2000 A) and low transverse emittance $(<2 \pi \mathrm{mm} \mathrm{mrad})$.

It is clear from Fig. 3 that $\mathrm{DC}$ and $\mathrm{LaB}_{6}$ cathode materials can satisfy the required $J_{\min }$ before reaching their melting temperatures. At first glance, DC seems the preferred candidate material due to its low temperature compared with $\mathrm{LaB}_{6}$ operating at the same current density. On the other hand, the change of current density during a $6 \mu \mathrm{s}$ macropulse is 6 times higher than $\mathrm{LaB}_{6}$ as shown in Fig. 5(b). The high change in the DC current density can introduce a very fast decrease in the electron beam energy. Moreover, from the experience of replacing the $6 \mathrm{~mm} \mathrm{DC}$ cathode with $2 \mathrm{~mm} \mathrm{LaB}_{6}$ in KU-FEL, $\mathrm{LaB}_{6}$ achieves a larger average beam current (120 mA compared to $70 \mathrm{~mA}$ for DC) at the same cavity field of $40 \mathrm{MV} \mathrm{m}^{-1}$ [24]. From the above arguments we suggest that a large change in current density for a thermionic cathode is not preferable in terms of BB effect.

It is well known that small transverse emittance is crucial for efficient FEL operation. From this point of 
TABLE III. Transverse emittance of $\mathrm{DC}$ and $\mathrm{LaB}_{6}$ cathodes.

\begin{tabular}{lcc}
\hline \hline & $\mathrm{DC}$ & $\mathrm{LaB}_{6}$ \\
\hline$T(\mathrm{~K})$ & 1476 & 1981 \\
$\varepsilon_{\mathrm{rms}}(\pi \mathrm{mm} \mathrm{mrad})$ & 0.249 & 0.289 \\
\hline \hline
\end{tabular}

view and the results shown in Table III, DC has priority as a cathode material compared with $\mathrm{LaB}_{6}$. The small emittance for DC is due to the small operation temperature for the cathode. However, the change in the DC temperature is higher than for $\mathrm{LaB}_{6}$ as shown in Fig. 5(a). From the above arguments, we can say that low operating temperature for a thermionic cathode is not preferable, in terms of changing the cathode temperatures.

The calculated stopping range and deposited heat power for BB electrons indicates that DC has short stopping range and high heat deposition compared to $\mathrm{LaB}_{6}$ as shown in Figs. 4(a) and 4(b), respectively. The short stopping range and the high deposited heat can be related to the high density for DC $\left(15.44 \mathrm{~g} \mathrm{~cm}^{-3}\right)$ compared to $\mathrm{LaB}_{6}$ $\left(4.72 \mathrm{~g} \mathrm{~cm}^{-3}\right)$. This means, interaction of BB electrons with dense materials deposits much higher heat than for low density materials. It can be seen from Fig. 4(c) that the deposited energy, as an example, from $100 \mathrm{keV} \mathrm{BB}$ electrons is $10 \mathrm{keV}_{\mu} \mathrm{m}^{-1}$ for $\mathrm{DC}$, about 5 times higher than for $\mathrm{LaB}_{6}\left(2 \mathrm{keV} \mu \mathrm{m}^{-1}\right)$. Thus, from the point of view of stopping range and deposited heat power $\mathrm{LaB}_{6}$ experiences only small effects due to $\mathrm{BB}$ electrons.

The numerical calculations for BB electrons in Figs. 5(a) and 5 (b) show a $\sim 106$ and $\sim 63 \mathrm{~K}$ increase of the cathode temperature for $\mathrm{DC}$ and $\mathrm{LaB}_{6}$ respectively, with an $\sim 87 \mathrm{~A} \mathrm{~cm}^{-2}$ and $\sim 15 \mathrm{~A} \mathrm{~cm}^{-2}$ increase in the respective current densities during the $6 \mu \mathrm{s}$ pulse. The "emission slope" can be defined as a change of the current density per change in a cathode temperature $\Delta J / \Delta T\left(\mathrm{~A} \mathrm{~cm}^{-2} \mathrm{~K}^{-1}\right)$ and is calculated to be $82 \%$ and $24 \%$ for $\mathrm{DC}$ and $\mathrm{LaB}_{6}$, respectively. This means the change in cathode temperature in DC is producing change of the current density much higher than for $\mathrm{LaB}_{6}$ and this can be related to the material characterizations.

The increase of the rf cavity beam loading is a direct result of the current density increase during the macropulse, and thus the cavity voltage decrease. Therefore, the expected decrease of beam energy in DC is much higher than in the $\mathrm{LaB}_{6}$ cathode under the same conditions. This can explain the improvement of the rf gun performance and extend of the macropulse up to $6 \mu$ s with stable electron beam energy after the replacement of the cathode material.

From comparison of simulation with experimental data for the $\mathrm{LaB}_{6}$ cathode beam current measured by CT1, the experimental data is well reproduced by simulation results as shown in Fig. 6(b).

From simulation results and argument, we have thus clarified the BB phenomenon, showing clearly that the
BB effect in the DC-based thermionic rf gun is much more serious than when using the $\mathrm{LaB}_{6}$ cathode.

\section{CONCLUSION}

We compared the BB effect for dispenser tungsten-base and lanthanum hexaboride cathodes to clarify the phenomenon and explain the improvement of KU-FEL thermionic $\mathrm{rf}$ gun performance after substitution of DC with $\mathrm{LaB}_{6}$. The first part of the analysis involved characterizing the electron beam emission properties. Stopping range and deposited heat power were then determined for BB electrons of various energies. The change of cathode temperatures and equivalent current densities were also determined numerically. Based upon the findings of this investigation, the $\mathrm{BB}$ effect for $\mathrm{DC}$ is much more detrimental than for the $\mathrm{LaB}_{6}$ cathode, having a much more significant effect on the cathode temperature and current density. Verification of the simulation model was also performed, with simulation results reproducing experimental data very well.

\section{ACKNOWLEDGMENTS}

The author wishes to thank the GCOE program, Graduate School of Energy Science in the age of Global Warming, Kyoto University, for financial support.

[1] J. Gao, Nucl. Instrum. Methods Phys. Res., Sect. A 297, 335 (1990).

[2] J. Gao, Rev. Sci. Instrum. 63, 64 (1992).

[3] S. V. Benson et al., Nucl. Instrum. Methods Phys. Res., Sect. A 250, 39 (1986).

[4] J.S. Fraser et al., in Proceedings of the 1987 Particle Accelerator Conference (IEEE, Washington, DC, 1987), p. 1705.

[5] K. Masuda et al., in 27th FEL 2005 Proceedings, eConf C0508213, 588 (2005).

[6] C. B. McKee and J. M. J. Madey, Nucl. Instrum. Methods Phys. Res., Sect. A 296, 716 (1990).

[7] T. Kii et al., Nucl. Instrum. Methods Phys. Res., Sect. A 483, 310 (2002).

[8] J.W. Lewellen, in Proceedings of the 20th Particle Accelerator Conference, Portland, OR, 2003 (IEEE, New York, 2003), p. 2035.

[9] T. Tanaka et al., in Proceedings of the 21st Particle Accelerator Conference, Knoxville, 2005 (IEEE, Piscataway, NJ, 2005), p. 3499.

[10] H. Zen et al., in Proceedings of the 11th European Particle Accelerator Conference, Genoa, 2008 (EPS-AG, Genoa, Italy, 2008), p. 1329.

[11] H. Zen et al., IEEE Trans. Nucl. Sci. 56, 1487 (2009).

[12] T. Kii et al., in Proceedings of Synchrotron Radiation Instrumentation: 9th International Conference on Synchrotron Radiation Instrumentation, AIP Conf. Proc. No. 879 (AIP, New York, 2007), pp. 248-251

[13] V. A. Kushnir, Probl. Atom. Sci. Technol. 3, 3 (1999); Nucl. Phys. Invest. 34, 3 (1999). 
[14] J. L. Cronin, IEE Proc. 128, 1 (1981).

[15] J. M. Lafferty, J. Appl. Phys. 22, 299 (1951).

[16] H. Kobayashi et al., in Proceedings of the 1992 Linear Accelerator Conference, Ottawa (1992), Vol. 1, p. 341.

[17] T. Tabata, R. Ito, and S. Okaba, Nucl. Instrum. Methods 103, 85 (1972).

[18] H. Ohgaki et al., in Proceedings of the 2007 Particle Accelerator Conference, Albuquerque, New Mexico (IEEE, New York, 2007), p. 254.
[19] N. Okawachi et al., in Proceedings of FEL2006 BESSY, Berlin Germany (2007), pp. 664-667.

[20] T. Shintake, Nucl. Instrum. Methods Phys. Res., Sect. A 363, 83 (1995).

[21] K. Masuda, Ph.D., thesis, Kyoto University, 1998.

[22] L. M. Young and J. H. Billen, LANL Report No. LA-UR96-1835 (2002).

[23] H. Ohgaki, J. Appl. Phys. 47, 8091 (2008).

[24] S. Sasaki et al., in the Proceedings of FEL2007, Novosibirsk, Russia (2008), pp. 394-397. 